\title{
Studying Enhanced Recovery After Surgery (ERAS®) Core Items in Colorectal Surgery: A Causal Model with Latent Variables
}

\author{
Marco Gemma ${ }^{1}$ Fulvia Pennoni ${ }^{2} \cdot$ Marco Braga $^{3}$
}

Accepted: 9 December 2020/Published online: 11 February 2021

(C) The Author(s) 2021

\begin{abstract}
Background Previous Enhanced Recovery After Surgery (ERAS $®$ ) studies have not always taken into account that ERAS interventions depend on baseline covariates and that several confounding variables affect the composite outcomes.

Method A causal latent variable model is proposed to analyze data obtained prospectively concerning 1261 patients undergoing elective colorectal surgery within the ERAS protocol. Primary outcomes (composite of any complication, surgical site infection, medical complications, early ready for discharge (TRD), early actual discharge) and secondary outcomes (composite of late bowel function recovery, IV fluid resumption, nasogastric tube replacement, postoperative nausea and vomiting, re-intervention, re-admission, death) are considered along with their multiple dimensions.

Results Concerning the primary outcomes, our results evidence three subpopulations of patients: one with probable good outcome, one with possibly prolonged TRD and discharge without complications, and the other one with probable complications and prolonged TRD and discharge. Epidural anesthesia, waiving surgical drainage, and early ambulation, IV fluid stop and urinary catheter removal act favorably, while preoperative hospital stay and blood transfusion act negatively. Concerning the secondary outcomes our results evidence two subpopulations of patients: one with high probability of good outcome and one with high probability of complications. Epidural anesthesia, waiving surgical drainage, early ambulation and IV fluid stop act favorably, while blood transfusion acts negatively also with respect to these secondary outcomes.

Conclusion The multivariate causal latent class two-parameter logistic model, a modern statistical method overcoming drawbacks of traditional models to estimate the average causal effects on the treated, allows us to disentangle subpopulations of patients and to evaluate ERAS interventions.
\end{abstract}

Fulvia Pennoni

fulvia.pennoni@unimib.it

Marco Gemma

marco.gemma@asst-fbf-sacco.it

1 Anesthesia and Intensive Care, Fatebenefratelli Hospital, Piazza Principessa Clotilde, 3, 20121 Milan, Italy

2 Department of Statistics and Quantitative Methods, University of Milano-Bicocca, Via degli Arcimboldi, 8, 20126 Milan, Italy

3 Department of Surgery, University of Milano-Bicocca, San Gerardo Hospital, Via Pergolesi G. B., 33, 20900 Monza, Italy

\section{Introduction}

The ERAS (Enhanced Recovery After Surgery) is a multimodal perioperative care pathway intended to improve and shorten recovery after major surgery through the application of a bundle of interventions [1, 2]. However implementing all of the ERAS items is a hard work for any hospital and it is possible that some ERAS interventions exert a greater impact on outcome than others. Although the final goal is to realize a complete ERAS pathway, concentrating on some possible "core items" in the 
beginning could be prominently facilitating. Much interest is growing about the search for evidence on these core items [3].

Any study on this topic exploited the incomplete compliance with ERAS items, which variably accompany ERAS databases and provides the necessary variability for addressing the question of the benefit of a single item or of ERAS as a whole. Nevertheless, previous analysis did not adequately consider that non-compliance is scarcely ever independent from other important variables. This raises at least three major methodological issues that have been poorly addressed in previous studies. First, ERAS outcomes and ERAS interventions themselves are affected by several confounding variables. For example, the American Society of Anesthesia score (ASA) status can affect both the outcome and the early postoperative mobilization. Second, ERAS items are themselves inter-related. For example, conservative intraoperative fluid administration is conceivably more applicable in patients who did not receive preoperative bowel preparation. Third, when dealing with ERAS, the outcome measures are composite. For example, the principal outcome measures in ERAS studies, postoperative length of hospital stay and complications, cannot be considered separately since they measure a similar trait.

We retrospectively studied a prospectively collected data of patients undergoing elective colorectal surgery between 2014 and 2018 with an ERAS protocol in 20 Italian hospitals affiliated with the PeriOperative Italian Society (POIS). The aim of our study is to analyze the effects of a number of ERAS items with a statistical model that considers the aforementioned methodological issues, namely a multivariate Latent Class Two-Parameter Logistic (LC-2PL) model [4, 5] formulated within a potential outcome framework [6]. We estimate the Average Causal Effects on the Treated (ATET) adequately weighting each patient through the Inverse-Probability-of-Treatment (IPT, [7]). As recently remarked by [8] propensity score methods allow us to block the association between observed confounding variables and treatments, thus permitting to reduce bias due to pre-treatment imbalances in observational studies. Innovatively we propose to estimate patient's weights according to the pre-treatment covariates and sequential blocks of treatments. We summarize the responses by means of a multivariate latent variable model suitable to classify patients and to assess the effects of the ERAS interventions on primary and secondary outcomes.

\section{Materials and methods}

\section{Study design}

Twenty Italian hospitals affiliated with POIS collaborated in collecting data. All centers treated their patients within an ERAS pathway, which was defined with active contribution from the ERAS Society. Before the start of the study, all hospitals had been involved in a pathway implementation program led by POIS consisting in education and audit meetings every six months for a two-year period.

All data were collected prospectively through a standardized electronic spreadsheet, which was used to record 90 variables per patient [9]. Every three months, the centerspecific spreadsheets containing data collected in that time period were merged into a web-based password-protected database managed by POIS. Data collected included demographics, patient comorbidities, preoperative and intraoperative variables, adherence to ERAS items, early recovery variables, and short-term postoperative outcomes.

Figure 1 shows the conceptual framework used to define sequentially the treatments, the confounders, the potential outcomes, the latent variables and the observed outcomes. We study 18 items out of the POIS database. For most of these treatments ERAS recommendations are available. Since our purpose is to account for the aforementioned inter-relationship between these treatments, we grouped them according to the phase of the patient's pathway in which they are applied. Hence, treatments are classified in three consecutive units, namely preoperative, intraoperative, and postoperative, and each unit affects the ones to follow. Four treatments are considered as preoperative, namely preoperative hospital stay (number of days), no bowel preparation, glucidic drink administration, and premedication. Six are considered as intraoperative, namely IV fluid administration $(\mathrm{ml} / \mathrm{kg} / \mathrm{h})$, epidural anesthesia, antibiotic prophylaxis, maintenance of normothermia, nausea and vomiting (PONV) prophylaxis, and no surgical drainage. Eight treatments are considered as postoperative, namely intravenous fluid administration $(\mathrm{ml} /$ $\mathrm{kg}$ during POD 1), morphine administration (dichotomous no/yes variable without differentiating between different administration modes-PCA, elastomer, fixed-dose-or different dosing), thromboembolism prophylaxis, prokinetic administration, naso-gastric tube (NGT) removal within POD 0, intravenous fluid stop within POD 2, urinary catheter removal within POD 1, and ambulation within POD 1. The cut-off POD choice for the last four variables has been decided according to the clinical experience.

There are 15 observed confounders. Seven of these potentially affect compliance with all of the treatments: 


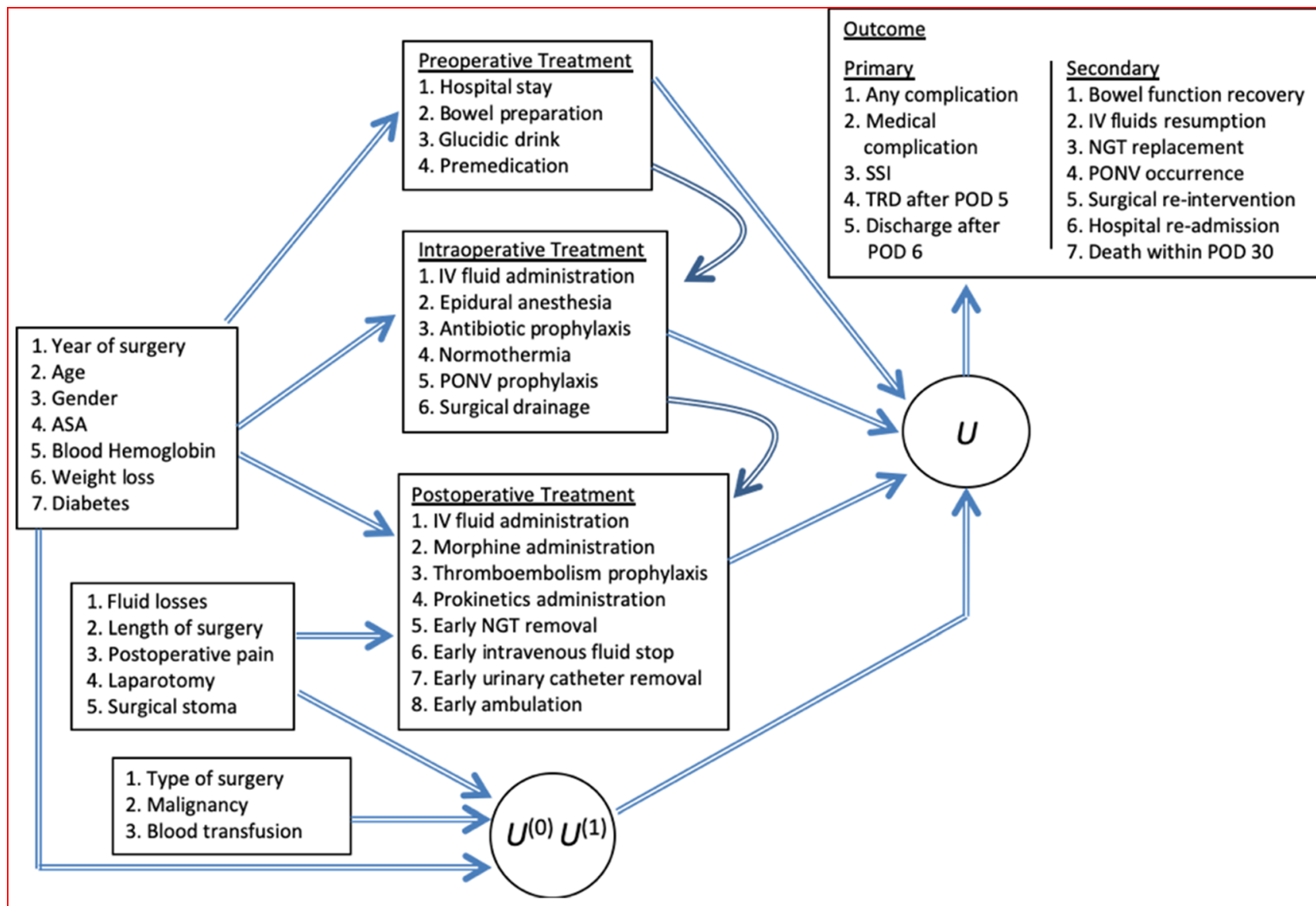

Fig. 1 Conceptual framework of the proposed multivariate causal latent variable model. IV: intravenous; NGT: nasogastric tube; PONV: postoperative nausea and vomiting; ASA: American Society of Anesthesia score; SSI: surgical site infection; TRD: time ready for discharge. U: latent variable; $U^{(0)}$ : latent potential outcome referred to the non-treated patient; $U^{(1)}$ : latent potential outcome referred to the treated patient

year of surgery, age, gender, ASA classification of general health status (ASA1-2 vs ASA > 2), baseline blood hemoglobin $(\mathrm{mg} / \mathrm{dl})$, preoperative body weight loss, and preoperative diagnosis of diabetes mellitus. Five confounders potentially affect compliance only with postoperative treatments: intraoperative fluid losses $(\mathrm{ml} / \mathrm{kg})$, length of surgery (min), maximum postoperative pain on POD 1-4 [measured on the Numerical Rating Scale (NRS)], laparotomy (vs laparoscopy), and surgical stoma (coded as binary variables). We also consider the following external variables that directly affect the outcomes: type of surgery (colonic or rectal), malignancy of the underlying disease, blood derivatives transfusion.

The main advantage of the proposed causal latent class model is that we jointly account for several outcomes that are distinguished as primary and secondary according to their relative clinical importance in the ERAS framework. The primary outcomes are the following: occurrence of any complication, occurrence of surgical site infection (SSI), occurrence of medical complications unrelated to the surgical site, such as cardiovascular, pulmonary, thromboembolic, or urinary complications, ready for discharge after POD 5, and actual discharge from hospital after POD 6 [10]. Within our proposal, as illustrated in the next section, we are able to consider the outcomes jointly and to account for the fact that they mainly concern two dimensions: the first is made by complications and the second one is made by Time Ready for Discharge (TRD) and actual discharge.

The secondary outcomes that we account jointly are the following: bowel function recovery after POD 1 , need for IV fluid resumption after suspension, need for nasogastric tube (NGT) replacement after removal, occurrence of postoperative nausea and vomiting (PONV), surgical reintervention, hospital re-admission, death within POD 30. In the multivariate model presented below we assume they represent two distinct dimensions: the first made by bowel function, need for IV fluid resumption, need for nasogastric tube, nausea and vomiting and the second made by surgical re-intervention, hospital re-admission and death. 


\section{Statistical model}

We highlight a proposal for the estimation of multiple treatments within observational studies, since we are interested to disentangle the effects of the ERAS items on primary and secondary outcomes. The treatment is confounded with the patient's characteristics and to address this problem we follow the potential outcome approach to causal inference as proposed by $[11,12]$ and we extend the IPT weighed estimator $[13,14]$ to a multivariate LC-2PL model [15]. The estimation of the probability to be treated is made sequentially along pre-treatment covariates and blocks of treatments (see Fig. 1).

Differently from previous proposals we suppose the counterfactual outcomes as latent variables denoted as $U_{i}^{(0)}$ and $U_{i}^{(1)}$ indicating for each patient $i, i=1, \ldots, n$, the variable under the non-treated and treaded status, respectively. An underlying latent variable $U_{i}$ is assumed to depend on both $U_{i}^{(0)}$ and $U_{i}^{(1)}$ as well as on the treatments (see Fig. 1). We assume local independence [5, 16] meaning that the observed outcomes are conditionally independent given the potential latent variables and the treatments administered sequentially.

In what follows, first we describe the estimation of the probability of treatment exposure given the pre-treatment covariates and each block of ERAS items (according to the arrows depicted in Fig. 1). This estimation is made by a sequence of linear or logistic regressions. Next, we calculate weights for each patient as the inverse of the probability of the observed treatment sequence. Third, we use stabilized weights to estimate a weighted causal LC-2PL model on the primary and secondary outcomes. The estimation is carried out through the maximization of a weighted log-likelihood employing the Expectation-Maximization algorithm (EM [17]). We rely on the Bayesian Information Criterion [BIC 18] through which we choose the suitable number of latent components. Fourth, we estimate the selected model by adding the covariates through a convenient parameterization considering the first latent class as reference since it identifies the subpopulation of patients recovered as expected.

The latent class model $[4,5]$ first proposed by [19] to classify units within a probabilistic approach is formulated as a finite mixture model [20]. Following some recent proposals in the literature, we formulate a multivariate LC2PL model [21] to infer causal effects within observational studies [15, 22, 23]. We introduce a novel use of this model by attempting to estimate the effects of various sequential interventions on patients entered in the ERAS project. We model the marginal distribution of the counterfactual variables $[6,24,25]$ and as a result the estimated regression coefficient encode the magnitude of the ATET [8]. In this way we mimic an artificial random assignment scenario essential to account for differences among patients. As pointed out by [7] the use of the Inverse-Probability-ofTreatment Weighting [IPTW 26] allow us to disentangle the association between the observed confounding variables and treatments thus permitting to reduce bias due to confounding.

\section{A causal latent variable model}

The potential outcomes of the patient are usually referred to as $Y_{i}^{(1)}$ if the patient is exposed to treatment $Z_{i}$ and as $Y_{i}^{(0)}$ if the patient is not exposed with $i, i=1, \ldots, n$. The treatment effect is given by the difference $Y_{i}^{(1)}-Y_{i}^{(0)}$ and the expected value of this difference over the entire population of treated patients is defined as the ATET. We instead postulate the existence of the underlying latent potential variables denoted as $U_{i}^{(z)}$ and we define the ATET as

$\mathrm{ATET}=\mathrm{E}\left(U_{i}^{(1)}-U_{i}^{(0)} \mid Z_{i}=1\right)$,

for $i, i=1, \ldots, n$, and of the latent variable $U_{i}$ depending on the treatment through the latent potential variables as follows

$U_{i}=\left(1-Z_{i}\right) U_{i}^{(0)}+Z_{i} U_{i}^{(1)}$.

For this latent variable we assume a discrete distribution left unspecified with a finite number of support points ranging from 1 to $k$.

Let $Y_{i r}$ be the observed binary response referred to outcome $r, r=1, \ldots, p$ for each patient $i, i=1, \ldots, n$, randomly drawn from the population. Following [27, 28] we extend the proposal of [15] to estimate patient specific weights. We formulate the following assumptions: $i$ ) conditional exchangeability meaning that the latent potential outcomes are independent on the treatment given the covariates, ii) positivity (ignorable treatment assignment) meaning that there is a positive probability for every patient of receiving any type of treatment, iii) consistency implying that the latent potential variables are well-defined and as a result any observed outcome is the potential variable corresponding to the observed treatment sequence.

Let $\boldsymbol{x}_{i}$ denotes the covariates for patient $i$ observed prior to the treatment assignment, the weight for this patient corresponds to the inverse of the conditional probability of receiving the treatment. In the case of a binary treatment we use the following logit model to estimate this probability 
$p_{i}=\log \frac{P\left(Z_{i}=1 \mid \boldsymbol{x}_{i}\right)}{P\left(Z_{i}=0 \mid \boldsymbol{x}_{i}\right)}=\alpha+\boldsymbol{x}_{i}^{\prime} \gamma$,

where $\alpha$ and $\gamma^{\prime}$ denotes the intercept and the vector of regression coefficients respectively. The weights are estimated sequentially according to the blocks of the ERAS items illustrated in Fig. 1. The arrows reported in this figure pointing from the risk factors into the other blocks indicate that the treatment is confounded and causally endogenous. The overall weight of patient $i$ is determined as the sum of the product of the estimated inverse-probabilities of the treatments in each block as follows

$\hat{w}_{i}=\sum_{v=1}^{V} \frac{1}{\prod_{j=1}^{J} p_{i j}}$,

where $v=1, \ldots, V$ denotes the block and $j, j=1, \ldots, J$ denotes the treatment in each block. The weights are stabilized by trimming them up to certain level to avoid high variability [23].

The dependence of the potential latent variables is modelled through the following multinomial logit model

$\log \frac{p\left(U_{i}^{(z)}=u\right)}{p\left(U_{i}^{(z)}=u-1\right)}=\beta_{0 u}+\boldsymbol{d}(z)^{\prime} \boldsymbol{\beta}_{1 u}$

where $u=2, \ldots, k, \beta_{0 u}$ is the intercept specific of each latent class, $\boldsymbol{d}(z)$ is a vector with elements equal to 1 for treated patients and $\boldsymbol{\beta}_{1 u}$ is the vector of parameters that define the ATET in the distribution of the latent variables.

Another set of parameters is referred to the conditional distribution of the observed outcomes and it is defined as

$p\left(Y_{i r}=1 \mid U_{i}^{(z)}=u\right)=\frac{1}{1+\exp \left[-\eta_{r}\left(\xi_{i}-\delta_{r}\right)\right]}$,

where $u=1, \ldots, k, \eta_{r}$ is a parameter measuring the discriminating power of the outcome $r, r=1, \ldots, p, \delta_{r}$ is another parameter measuring the difficulty of the outcome, and $\xi_{i}$ indicates the point on the latent continuum where patient $i, i=1, \ldots, n$ is located. This is a $2 \mathrm{PL}$ model specification for the dichotomously scored outcomes.

The model is estimated through a weighted log-likelihood function by considering a sample of $n$ independent patients for which we observe the multivariate binary outcomes. The target log-likelihood function is

$l(\boldsymbol{\theta})=\sum_{i=1}^{n} \hat{w}_{i} l_{i}(\boldsymbol{\theta})$,

where $\boldsymbol{\theta}$ denotes the overall vector of free parameters arranged in a suitable way. This weighted log-likelihood is maximized through the EM algorithm [17]. The latter estimates the model parameters considering as missing data the vector of latent variables. Then E-step of the algorithm computes the conditional expected value of the completedata log-likelihood given the observed data and the current value of the parameters. The M-step updates the parameters maximizing the expected value of the quantity computed at the E-step. The two steps are alternated repeatedly until a convergence criterion is reached.

In order to choose the best number of latent classes and to discover meaningful groups of patients in the population we apply a model selection strategy. The resulting subpopulations should be internally cohesive and well separated from one another. We rely on the BIC index [18] that is a measure of the relative goodness of fit of the model able to account simultaneously for its accuracy and complexity. It is defined as

$\mathrm{BIC}=-2 l(\hat{\boldsymbol{\theta}})+\log (n) \#$ par,

where $l(\hat{\boldsymbol{\theta}})$ denotes the maximum of the weighted loglikelihood of the model with $k$ latent classes, \#par denotes the number of free parameters and $n$ is the sample size. The model is estimated for an increasing number of latent classes and the best model is selected as that before the BIC starts to increase. Once the suitable number of latent classes is selected every patient is allocated to a latent class according the highest posterior probability. Standard errors for the estimated parameters are obtained as the square root of the diagonal elements of the inverse of the observed or expected information matrix computed through numerical methods.

\section{Results}

The available observations are related to 1261 patients operated between 2014 and 2018. Table 1 shows some descriptive statistics. The model is estimated by using the open source software $R$ [29] through the package multiLCIRT [30]. As far as we know there are no other software able to account for the multidimensionality of the responses and latent variables with a discrete distribution. For replicability purposes the code to estimate the proposed model is available from the repository at the following link https://github.com/penful/Eras. The complete results are available from the authors upon request.

\section{Results for the primary outcomes}

The multivariate causal LC-2PL model is estimated for a number of latent classes ranging from 1 to 5 . According to the lowest value of the BIC index we select the model with three latent classes. Table 2 reports on the estimated conditional probabilities of the primary outcomes. The first class, which encompasses $47 \%$ of the patients, presents low 
Table 1 Frequency distribution of the available data

\begin{tabular}{|c|c|}
\hline \multicolumn{2}{|l|}{ Characteristics } \\
\hline Colon (vs rectal) surgery & $962(76.29)$ \\
\hline Malignant lesion & $1058(83.90)$ \\
\hline Blood transfusion & $116(9.20)$ \\
\hline \multicolumn{2}{|l|}{ Preoperative treatment } \\
\hline Preoperative hospital stay (days) & $1.56 \pm 3.56$ \\
\hline Bowel preparation & $160(12.69)$ \\
\hline Glucidic drink & $887(70.34)$ \\
\hline Premedication & $522(41.40)$ \\
\hline \multicolumn{2}{|l|}{ Intraoperative treatment } \\
\hline IV fluids $(\mathrm{ml} / \mathrm{kg} / \mathrm{h})$ & $8.95 \pm 4.43$ \\
\hline Epidural anesthesia & $468(37.11)$ \\
\hline Antibiotic prophylaxis & $1243(98.57)$ \\
\hline Maintenance of normothermia & $1243(98.57)$ \\
\hline PONV prophylaxis & $940(74.54)$ \\
\hline Surgical drainage & $862(68.36)$ \\
\hline \multicolumn{2}{|l|}{ Postoperative treatment } \\
\hline IV fluids on POD 1 (ml/kg) & $21.57 \pm 11.24$ \\
\hline Morphine & $458(36.32)$ \\
\hline Thrombosis prophylaxis & $1250(99.13)$ \\
\hline Prokinetics & $368(29.18)$ \\
\hline Removal of NGT (POD) & $0.16 \pm 0.73$ \\
\hline Stop of IV fluids (POD) & $2.52 \pm 2.52$ \\
\hline UC removal (POD) & $1.74 \pm 1.96$ \\
\hline Ambulation within POD 1 & $1043(82.71)$ \\
\hline \multicolumn{2}{|l|}{ Covariates } \\
\hline Age (yrs) & $67.25 \pm 11.78$ \\
\hline Male gender & $676(53.61)$ \\
\hline ASA $>2$ & $441(34.97)$ \\
\hline Baseline serum hb (mg/dl) & $12.86 \pm 1.98$ \\
\hline Preoperative weight loss & $84(6.66)$ \\
\hline Diabetes mellitus & $162(12.85)$ \\
\hline Intraoperative fluid loss (ml/kg) & $4.81 \pm 2.60$ \\
\hline Length of surgery (min) & $203.26 \pm 75.63$ \\
\hline Max pain on POD 1-4 (NRS) & $3.55 \pm 2.02$ \\
\hline Laparotomy & $251(19.90)$ \\
\hline Surgical stoma & $192(15.23)$ \\
\hline \multicolumn{2}{|l|}{ Primary outcomes } \\
\hline Any complication & $259(20.54)$ \\
\hline Medical complication & $67(5.31)$ \\
\hline SSI & $91(7.22)$ \\
\hline TRD after POD 5 & $6.26(4.75)$ \\
\hline Discharge after POD 6 & $6.6(4.64)$ \\
\hline \multicolumn{2}{|l|}{ Secondary outcomes } \\
\hline Bowel function recovery (POD) & $1.9(1.11)$ \\
\hline IV fluids resumption & $158(12.53)$ \\
\hline NGT replacement & $96(7.61)$ \\
\hline PONV occurrence & $133(10.55)$ \\
\hline Surgical re-intervention & $59(4.68)$ \\
\hline
\end{tabular}

Table 1 continued

\begin{tabular}{ll}
\hline Characteristics & \\
\hline Hospital re-admission & $23(1.82)$ \\
Death within POD 30 & $7(0.56)$
\end{tabular}

ASA: American Society of Anesthesia status score; PONV: postoperative nausea and vomiting; POD: postoperative day; NGT: nasogastric tube; SSI: surgical site infection; hb: serum hemoglobin; IV: intravenous; UC: urinary catheter; TRD: time ready to discharge. Categorical variables are reported as number (percentage), continuous variables as mean \pm standard deviation. The year of the surgery is omitted from the table

Table 2 Estimated probability of each latent class and estimated conditional probabilities of the multivariate causal latent class twoparameter logistic model for the primary outcomes

\begin{tabular}{lccc}
\hline & \multicolumn{3}{c}{ Latent class } \\
\cline { 2 - 4 } & 1 & 2 & 3 \\
\hline Estimated probability of each Latent Class & 0.47 & 0.35 & 0.18 \\
Estimated conditional probability for primary & outcomes & \\
Any complication & 0.07 & 0.08 & 0.82 \\
SSI & 0.01 & 0.01 & 0.35 \\
Medical complication & 0.02 & 0.02 & 0.28 \\
TRD after POD 5 & 0.00 & 0.94 & 0.95 \\
Discharge after POD 6 & 0.00 & 0.65 & 0.87 \\
\hline
\end{tabular}

SSI: surgical site infection; TRD: time ready for discharge; POD: postoperative day. Any complications, SSI and medical complication measure the first dimension, TRD after POD 5 and Discharge after POD 6 measure the second dimension

probability of complications and of late discharge. The second class, representing $35 \%$ of the patients, exhibits a high probability of late TRD and actual discharge although complications are improbable. Patients in the third class, accounting for the remaining $18 \%$ of patients, present high probability of complication and late TRD and actual discharge. Since these subpopulations are ordered according to the outcome occurrence we define the first LC as that representing the subpopulation of the best performing patients, the second that of intermediate patients and the third that of worst performing patients.

By looking at the estimated ATETs defined with respect to the first LC chosen as reference due to the fact that it collects patients with the best outcomes, we evaluate the efficacy of each intervention. Table 3 reports on the estimated ATETs of being in the 1st rather than in the 2nd LC. The estimated regression coefficients in the upper part of 
Table 3 Estimated effects of the causal latent class two-parameter logistic model for being in the 1st latent class rather than in the 2nd latent class for the primary outcomes. Estimated standard errors and asymptotic confidence interval at confidence level of 0.95

\begin{tabular}{|c|c|c|c|c|}
\hline 1st versus 2nd Latent Class & Estimated coefficient & S.E. & CI & \\
\hline Intercept & 1.49 & 1.08 & $(-0.63,3.61)$ & \\
\hline No bowel preparation & $-0.85^{*}$ & 0.51 & $(-1.21,-0.43)$ & Favor being in the 1 st $\mathrm{LC}$ \\
\hline Colon surgery & $-0.82 * * *$ & 0.20 & $(-1.85,0.15)$ & \\
\hline Ambulation within POD 1 & $-0.76^{* * *}$ & 0.21 & $(-1.17,-0.35)$ & \\
\hline IV fluid stop within POD 2 & $-0.75 * * *$ & 0.17 & $(-1.08,-0.42)$ & \\
\hline UC removal within POD 1 & $-0.62 * * *$ & 0.19 & $(-0.99,-0.25)$ & \\
\hline Epidural anesthesia & $-0.39 * *$ & 0.18 & $(-0.74,-0.04)$ & \\
\hline No surgical drainage & $-0.35^{*}$ & 0.18 & $(-0.70,0.00)$ & \\
\hline Prokinetics & -0.15 & 0.16 & $(-0.46,0.16)$ & \\
\hline NGT removed on POD 0 & -0.15 & 0.27 & $(-0.68,0.38)$ & \\
\hline Morphine & -0.05 & 0.16 & $(-0.36,0.26)$ & \\
\hline Preoperative glucidic drink & -0.04 & 0.16 & $(-0.35,0.27)$ & \\
\hline Intravenous fluids & -0.02 & 0.02 & $(-0.06,0.02)$ & \\
\hline IV fluids during POD 1 & 0.01 & 0.01 & $(-0.01,0.03)$ & Favor being in the 2 nd LC \\
\hline Preoperative hospital stay & $0.05 * *$ & 0.02 & $(0.01,0.09)$ & \\
\hline PONV prophylaxis & 0.08 & 0.19 & $(-0.29,0.45)$ & \\
\hline Premedication & 0.40 & 0.24 & $(-0.07,0.87)$ & \\
\hline Malignant lesion & $0.45 * *$ & 0.20 & $(0.06,0.84)$ & \\
\hline Thromboembolism prophylaxis & 0.69 & 0.90 & $(-1.07,2.45)$ & \\
\hline Blood transfusion & $1.16^{* *}$ & 0.32 & $(0.53,1.79)$ & \\
\hline
\end{tabular}

LC: latent class; IV: intravenous; UC: urinary catheter; NGT: nasogastric tube; POD: postoperative day. Significance levels for the test based on the estimated standard errors that each parameter is equal to zero: *significant at $1 \%$; **significant at $5 \%$; ***significant at $10 \%$

the table indicate that no bowel preparation, colon surgery, ambulation within POD 1, IV fluid stop within POD 2, urinary catheter removal within POD 1, epidural anesthesia, and not inserting any surgical drainage significantly favor being in the class of best performing patients (1st LC) rather than in the group of less (intermediate) performing patients (2nd LC).

The estimated regression coefficients in the bottom part of the table indicate that preoperative hospital stay, malignant lesion, and blood transfusion significantly favor being in the group of intermediate performing patient (2nd LC) with respect to best performing (1st LC).

Table 4 reports on the estimated ATETs of being in the 2nd rather than in the $3 \mathrm{rd} \mathrm{LC}$. The estimated regression coefficients in the upper part of the table indicate that ambulation within POD 1, epidural anesthesia, IV fluid stop within POD 2, urinary catheter removal within POD 1, and not inserting any surgical drainage favor being in the class of intermediate performing patients (2nd LC) rather than in the group of worst performing patients (3rd LC).

The estimated regression coefficients in the bottom part of the table indicate that postoperative fluids, preoperative hospital stay, PONV prophylaxis, premedication, and blood transfusion significantly favor being in the group of worst performing patients (3rd LC) rather than stay in the intermediate group of patients (2nd LC).

\section{Results for the secondary outcomes}

The multivariate causal LC-2PL model for the secondary outcomes is estimated as the previous model for a number of latent classes ranging from 1 to 5 . According to the lowest value of the BIC index we select the model with two latent classes. The estimates of the model parameters for the secondary outcomes are reported in Tables 5 and 6 . According to the results showed in Table 5 we notice that both latent classes have a similar probability of bowel function recovery after POD 1 ( 0.51 and 0.56 respectively) and a null or very low probability of death before POD 30 $(0.00$ and 0.01 respectively). The first class $(78 \%$ of patients) exhibits low probability for all the other secondary outcomes. In the second latent class IV fluid resumption, NGT replacement, PONV occurrence and surgical re-intervention are sensibly more probable, while hospital re-admission is only slightly more probable.

Table 6 reports on the estimated ATETs of being in the 1 st rather than in the 2 nd LC. The estimated regression coefficients in the upper part of the table indicate that 
Table 4 Estimated effects of the causal latent class two-parameter logistic model for being in the 2nd rather than in the 3rd latent class for the primary outcomes. Estimated standard errors and asymptotic confidence interval at confidence level of 0.95

\begin{tabular}{|c|c|c|c|c|}
\hline 2 st versus 3rd Latent Class & Estimated coefficient & S.E. & $\mathrm{CI}$ & \\
\hline Intercept & $2.08^{*}$ & 1.14 & $(-0.15,4.31)$ & \\
\hline Ambulation within POD 1 & $-1.51 * * *$ & 0.28 & $(-2.06,-0.96)$ & Favor being in the 2 nd LC \\
\hline Thromboembolism prophylaxis & -1.12 & 0.83 & $(-2.75,0.51)$ & \\
\hline Epidural anesthesia & $-0.90 * * *$ & 0.27 & $(-1.43,-0.37)$ & \\
\hline IV fluid stop within POD 2 & $-0.81 * * *$ & 0.24 & $(-1.28,-0.34)$ & \\
\hline UC removal within POD 1 & $-0.68 * *$ & 0.26 & $(-1.19,-0.17)$ & \\
\hline No surgical drainage & $-0.67 * *$ & 0.26 & $(-1.18,-0.16)$ & \\
\hline No bowel preparation & -0.48 & 0.72 & $(-1.89,0.93)$ & \\
\hline Colon surgery & -0.36 & 0.28 & $(-0.91,0.19)$ & \\
\hline Prokinetics & -0.31 & 0.25 & $(-0.80,0.18)$ & \\
\hline NGT removed on POD 0 & -0.08 & 0.36 & $(-0.79,0.63)$ & \\
\hline Intraoperative fluids & -0.04 & 0.03 & $(-0.10,0.02)$ & \\
\hline IV fluids during POD 1 & $0.02 * *$ & 0.01 & $(0.00,0.04)$ & Favor being in the 3 rd LC \\
\hline Preoperative hospital stay & $0.05 * *$ & 0.02 & $(0.01,0.09)$ & \\
\hline Preoperative glucidic drink & 0.20 & 0.22 & $(-0.23,0.63)$ & \\
\hline Morphine & 0.24 & 0.21 & $(-0.17,0.65)$ & \\
\hline Malignant lesion & 0.38 & 0.28 & $(-0.17,0.93)$ & \\
\hline PONV prophylaxis & $0.54 * *$ & 0.28 & $(-0.01,1.09)$ & \\
\hline Premedication & $0.97 * * *$ & 0.33 & $(0.32,1.62)$ & \\
\hline Blood transfusion & $1.90 * * *$ & 0.35 & $(1.21,2.59)$ & \\
\hline
\end{tabular}

LC: latent class; IV: intravenous; UC: urinary catheter; NGT: nasogastric tube; POD: postoperative day. Significance levels for the test according to the estimated standard errors that each parameter is equal to zero: *significant at $1 \%$; **significant at $5 \%$; ***significant at $10 \%$

thromboembolism prophylaxis, ambulation within POD 1, IV fluid stop within POD 2, NGT removal on POD 0, not inserting any surgical drainage, and epidural anesthesia favor being in the class of best performing patients (1st LC) rather than in the group of the worst performing patients (2nd LC).

The estimated ATETs in the bottom part of the table indicate that blood transfusion, preoperative glucidic drink, and prokinetics significantly favor being in the group of the worst performing patient (2nd LC) with respect to best performing (1st LC).

\section{Discussion}

With respect to our primary outcomes, the model points out three classes of patients. The first one has a high probability of good outcome and represents the majority of our patients. The second class exhibits possibly prolonged TRD and discharge notwithstanding the absence of complications. The third class presents high probability of both complications and prolonged TRD and discharge. Five variables positively affect the outcomes (ambulation within POD 1, IV fluid stop within POD 2, urinary catheter removal within
POD 1, epidural anesthesia, and not inserting any surgical drainage). Two variables negatively affect the outcomes (preoperative hospital stay and blood transfusion).

With respect to our secondary outcomes, three of them could not contribute to discrimination between classes of patients, since their probability was uniformly either low (hospital re-admission and death within POD 30) or high (bowel function recovery after POD 1). The model points out two classes of patients: a first class with high probability of good outcome (including the majority of our patients), and a second one with high probability of IV fluid resumption, NGT replacement, PONV occurrence, and surgical re-intervention. Six variables positively affect the secondary outcomes and four of them do the same for the primary outcomes (ambulation within POD 1, IV fluid stop within POD 2, epidural anesthesia, and not inserting any surgical drainage). Four variables negatively affect the secondary outcomes and one of them does the same for the primary outcomes (blood transfusion).

Our results contribute to the ongoing debate about the existence of ERAS "core-items". In fact, although it is recognized that the complete ERAS protocol is the best way to improve postoperative outcome, the number and relative combination of the ERAS items implemented in 
Table 5 Estimated probability of each latent class and estimated conditional probabilities of the multivariate causal latent class two-parameter logistic model for the secondary outcomes

\begin{tabular}{|c|c|c|c|}
\hline & & \multicolumn{2}{|c|}{ Latent class } \\
\hline & & 1 & 2 \\
\hline \multicolumn{2}{|l|}{ Estimated probability of each Latent Class } & 0.78 & 0.22 \\
\hline \multirow[t]{7}{*}{ Estimated conditional probability for secondary outcomes } & Bowel function recovery after POD 1 & 0.51 & 0.56 \\
\hline & IV fluid resumption & 0.03 & 0.57 \\
\hline & NGT replacement & 0.00 & 0.34 \\
\hline & PONV occurrence & 0.08 & 0.41 \\
\hline & Surgical re-intervention & 0.01 & 0.16 \\
\hline & Hospital re-admission & 0.00 & 0.07 \\
\hline & Death within POD 30 & 0.00 & 0.01 \\
\hline
\end{tabular}

POD: postoperative day; IV: intravenous; NGT: nasogastric tube; PONV: postoperative nausea and vomiting measure the first dimension and the remaining outcomes measure the second dimension

previous works varied across studies without substantial differences in postoperative short-term outcomes [31, 32]. Indeed, several studies imply that some ERAS elements may be more significant than others in affecting outcome [33-36] and that simplified protocols could yield comparable results $[23,24]$. A systematic review suggests that the number of implemented ERAS items does not affect outcome and that applying only some core-items is sufficient to obtain the benefits of the ERAS program [37]. In contrast, a large cohort of patients from the ERAS Society Registry suggested that the improvement in clinical outcome provided by an ERAS program was directly correlated with the number of implemented items and the degree of compliance to the protocol $[38,39]$.

In addressing these issues no previous study adequately considered the dependency of multivariate outcomes from confounding variables and non-compliance to ERAS items, the inter-relation between ERAS items themselves, and the composite nature of the primary and secondary outcomes at stake.

The estimation of treatment effects in observational studies when the treatment assignment depends on the sequence of previous assignments and on time-varying confounders is still a matter of debate. The main advantage over the standard simple multinomial logit model is that our counterfactual framework assesses causal associations, corrects for pre-treatment confounders and for multiple treatment conditions in order to reduce the bias due to confounding. Another advantage is that it is a multivariate model-based clustering method and allows us to properly account for suitable groups able to disentangle the heterogeneous population of patients. Moreover, it yields a classification that uses the maximum a-posteriori estimates of the model parameters.
The results of the application provide evidence that waiving bowel preparation increases the probability of good outcome. Recent ERAS guidelines on colonic surgery and a large meta-analysis of more than 21,000 patients agree that mechanical bowel preparation is not associated to any reduction in postoperative complications, mortality, and length of hospital stay when compared with no preparation [40, 41]. Actually, waiving preoperative mechanical bowel preparation reduces the risk of preoperative dehydration and the possibly associated electrolyte disorders. This favors a reduction in fluid administration and the reaching of zero fluid balance. Moreover, a possible increment in Gram-negative bacterial components of the intestinal flora is associated with bowel preparation [42].

Recent studies demonstrate how a balanced intraoperative goal directed therapy reduces mortality, overall morbidity, and the time to first flatus and to oral feeding [43]. The ERAS guidelines clearly state that postoperative IV fluid administration is not necessary if oral intake is possible and that early oral feeding is safe and well tolerated by the majority of patients after colorectal surgery [44]. Early stop of IV fluid infusion and urinary catheter removal, together with good pain control and no surgical drainage, foster patients' mobilization after surgery. A reduction of postoperative pulmonary and thromboembolic complications and a regain of preoperative functional capacity and muscular strength are strictly related to early postoperative mobilization [45].

The results suggest that epidural analgesia exerts a favorable effect on outcome and therefore they confirm the findings of other studies according to which epidural analgesia after laparotomy provides optimal pain control and reduces the inflammatory stress response. This reduces the incidence of pulmonary and cardiovascular 
Table 6 Estimated effects of the causal latent class two-parameter logistic model for being in the 1st latent class rather than in the 2nd latent class for the secondary outcomes. Estimated standard errors and asymptotic confidence interval at confidence level of 0.95

\begin{tabular}{|c|c|c|c|c|}
\hline 1 st vs 2 nd LC & Estimated coefficient & S.E. & $\mathrm{CI}$ & \\
\hline Intercept & 1.24 & 1.37 & $(-1.45,3.93)$ & \\
\hline Thromboembolism prophylaxis & $-2.56 * * *$ & 0.80 & $(-4.13,-0.99)$ & Favor being in the 1 st LC \\
\hline Ambulation within POD 1 & $-1.48 * * *$ & 0.27 & $(-2.01,-0.95)$ & \\
\hline IV fluid stop within POD 2 & $-1.45 * * *$ & 0.25 & $(-1.94,-0.96)$ & \\
\hline NGT removed on POD 0 & $-1.21 * * *$ & 0.36 & $(-1.92,-0.50)$ & \\
\hline No bowel preparation & -0.77 & 0.92 & $(-2.57,1.03)$ & \\
\hline No surgical drainage & $-0.48 *$ & 0.27 & $(-1.01,0.05)$ & \\
\hline Epidural anesthesia & $-0.47 *$ & 0.27 & $(-1.00,0.06)$ & \\
\hline Colon surgery & -0.23 & 0.28 & $(-0.78,0.32)$ & \\
\hline PONV prophylaxis & -0.13 & 0.28 & $(-0.68,0.42)$ & \\
\hline UC removal within POD 1 & -0.04 & 0.26 & $(-0.55,0.47)$ & \\
\hline Intraoperative fluids & -0.02 & 0.03 & $(-0.08,0.04)$ & \\
\hline Morphine & -0.02 & 0.23 & $(-0.47,0.43)$ & \\
\hline IV fluids during POD 1 & -0.01 & 0.01 & $(-0.03,0.01)$ & \\
\hline Preoperative hospital stay & 0.00 & 0.03 & $(-0.06,0.06)$ & Favor being in the 2 nd LC \\
\hline Malignant lesion & 0.04 & 0.30 & $(-0.55,0.63)$ & \\
\hline Premedication & 0.46 & 0.38 & $(-0.28,1.20)$ & \\
\hline Blood transfusion & $0.87 * *$ & 0.33 & $(0.22,1.52)$ & \\
\hline Preoperative glucidic drink & $0.97 * *$ & 0.28 & $(0.42,1.52)$ & \\
\hline Prokinetics & $1.22 * *$ & 0.20 & $(0.75,1.69)$ & \\
\hline
\end{tabular}

LC: latent class; IV: intravenous; UC: urinary catheter; NGT: naso-gastric tube; POD: postoperative day. Significance levels for the test based on the estimated standard errors that each parameter is equal to zero: *significant at $1 \%$; **significant at $5 \%$; ***significant at $10 \%$

complications, in particular in frail patient $[46,47]$. Nevertheless, the role of thoracic epidural analgesia after laparoscopic procedures is controversial and the importance of multimodal analgesic sparing-opioids strategies is widely accepted in less invasive surgery. Studies addressing purely laparoscopic colonic surgery suggest caution towards epidural analgesia [48]. It should be noted that, although laparoscopy is widely recognized as a predictor of faster recovery, this effect was not apparent in our results.

The negative effect of a prolonged preoperative hospital stay, which we observed, deserves attention. Serious comorbidities requiring longer preoperative hospitalization can make it difficult to optimize patients' conditions and preserve functional capacity before surgery. It has been recently demonstrated that a decline in preoperative functional capacity, determined by the Duke score activity index, is associated to increased myocardial infarction and death 30 days and one year after major non-cardiac surgery [49]. Prehabilitation, as preoperative optimization of patients' condition, is a key ERAS concept [50]. However, it is evident that this should not prolong the preoperative hospital stay. Our analysis supports the hypothesis that preoperative hospital stay may even impair prehabilitation. Our results on the adverse effect of blood transfusion on outcome support the evidence that a careful management of preoperative anemia through iron supplemental therapy can improve overall morbidity and mortality by reducing the need for blood transfusion in the perioperative period [39, 51].

A possible limitation of our study is related to conceivable differences between the participating hospitals and through the time span of the data collection, both in the degree of ERAS pathway implementation and in the baseline standard of care. A potential selection bias could be at stake, despite all centers have been invited to submit consecutive elective patients. Nevertheless, the wide mix of ages and comorbidities included indicates a small likelihood of selection bias among patients.

The major strength of our proposal resides in the features of the proposed statistical method. Moreover, we accessed a dedicated ERAS database from which we derived the data used for the analyses and we used a validated indicator of short-term recovery such as the TRD [10]. 


\section{Conclusion}

We analyze a colonic surgery ERAS database by proposing a multivariate causal latent class two-parameter logistic model. This modern statistical method overcomes several drawbacks of traditional methods to estimate average treatment effects on the treated. Since we are dealing with observational studies we employ a propensity score method. We propose to use a maximum likelihood approach by employing weights estimated through the inverse-probability of receiving the treatments. In this way, we avoid rough comparisons of patients thus producing a valid inference and reducing the possibility of biased treatment effects. As noted by [52] generally a simple covariate adjustment cannot be able to produce unbiased estimates of the model parameters. The proposed method of analysis is able to account for patient heterogeneity and it constitutes a general approach for the analysis of data arising in similar medical contexts.

According to the results early postoperative ambulation and IV fluid stop, epidural anesthesia, and waiving any surgical drainage exert a favorable effect on primary outcomes (time ready for discharge and actual discharge from hospital, and occurrence of any complication, surgical site infection, and medical complications), while prolonged preoperative hospital stay and blood transfusion act unfavorably.

Acknowledgements Turi S. ${ }^{1}$ MD, Monzani R. ${ }^{2}$ MD, Radrizzani D. ${ }^{3}$ MD, Beretta L. ${ }^{1}$ MD Scatizzi M. ${ }^{4}$ MD, Borghi F. ${ }^{5}$ MD, Missana G. ${ }^{6}$ MD, Azzola A. ${ }^{7}$ MD, Muratore A. ${ }^{8}$ MD, Crespi M. ${ }^{9}$ MD, Iuliani R. ${ }^{10}$ MD, Bima C. ${ }^{10}$ MD, Bouzari H. ${ }^{11}$ MD, Pisani Ceretti A. ${ }^{12}$ MD, Pellegrino L. ${ }^{5}$ MD, Casiraghi U. ${ }^{1}$, Ficari $F^{13}$ MD.

${ }^{1}$ Anesthesia and Intensive Care, IRCCS San Raffaele, Milan, Italy, ${ }^{2}$ Department of Anesthesia, Humanitas Research Hospital, Rozzano, Milan, Italy, ${ }^{3}$ Department of Anesthesia, Legnano Hospital, Milan, Italy, ${ }^{4}$ Department of Surgery, Prato Hospital, Prato, Italy, ${ }^{5}$ Department of Surgery, Cuneo Hospital, Cuneo, Italy, ${ }^{6}$ Department of Surgery, Casa di Cura Città di Udine, Udine, Italy, ${ }^{7}$ Department of Surgery, Cantù Hospital, Cantù, Italy, ${ }^{8}$ Department of Surgery, Candiolo Hospital, Turin, Italy, ${ }^{9}$ Department of Surgery, Luigi Sacco Hospital, Milan, Italy, ${ }^{10}$ Department of Surgery, Cottolengo Hospital Turin, Turin, Italy, ${ }^{11}$ Department of Surgery, Mauriziano Hospital Turin, Turin, Italy, ${ }^{12}$ Department of Surgery, San Paolo Hospital, Milan, Italy, ${ }^{13}$ Department of Surgery, Careggi Hospital, University of Florence, Florence, Italy

Funding Open Access funding provided by Università degli Studi di Milano - Bicocca.

Open Access This article is licensed under a Creative Commons Attribution 4.0 International License, which permits use, sharing, adaptation, distribution and reproduction in any medium or format, as long as you give appropriate credit to the original author(s) and the source, provide a link to the Creative Commons licence, and indicate if changes were made. The images or other third party material in this article are included in the article's Creative Commons licence, unless indicated otherwise in a credit line to the material. If material is not included in the article's Creative Commons licence and your intended use is not permitted by statutory regulation or exceeds the permitted use, you will need to obtain permission directly from the copyright holder. To view a copy of this licence, visit http://creativecommons. org/licenses/by/4.0/.

\section{References}

1. Ljungqvist O (2014) ERAS-enhanced recovery after surgery: moving evidence-based perioperative care to practice. J Parenter Enteral Nutr 38:559-566

2. Ljungqvist O, Scott M, Fearon KC (2017) Enhanced recovery after surgery: a review. JAMA Surg 152:292-298

3. Braga M, Scatizzi M, Borghi F et al (2018) Identification of core items in the enhanced recovery pathway. Clin Nutr Espen 25:139-144

4. Lazarsfeld PF, Henry NW (1968) Latent structure analysis. Houghton Mifflin, Boston

5. Pennoni F (2014) Issues on the estimation of latent variable and latent class models. Scholars Press, Saarbücken

6. Rubin DB (2005) Causal inference using potential outcomes: design, modeling, decisions. J Am Stat Assoc 100:322-331

7. Robins JM, Hernan M, Brumback B (2000) Marginal structural models and causal inference in epidemiology. Epidemiology 5:550-560

8. Schulte PJ, Mascha EJ (2018) Propensity score methods; theory and practice for anesthesia research. Anesth Analg 127:1074-1084

9. Braga M, Borghi F, Scatizzi M et al (2017) Impact of laparoscopy on adherence to an enhanced recovery pathway and readiness for discharge in elective colorectal surgery: results from the Perioperative Italian Society registry. Surg Endosc 31:4393-4399

10. Fiore JF, Faragher IG, Bialocerkowski A et al (2013) Time to readiness for discharge is a valid and reliable measure of shortterm recovery after colorectal surgery. World J Surg 37:2927-2934

11. Rubin DB (1974) Estimating causal effects of treatments in randomized and nonrandomized studies. J Educ Psychol 6:688-701

12. Holland PW, Rosenbaum PR (1986) Conditional association and unidimensionality in monotone latent variable models. Ann Statist 4:1523-1543

13. Greenland S, Robins JM (1986) Identifiability, exchangeability, and epidemiological confounding. Int J Epidemiol 15:413-419

14. Cole SR, Hernan MA (2008) Inverse probability weights for marginal structural models. Am J of Epidemiol 6:656-664

15. Lanza ST, Coffman DL, Xu S (2013) Causal inference in latent class analysis. Struct Equ Model 20:361-383

16. Bartolucci F, Farcomeni A, Pennoni F (2013) Latent Markov models for longitudinal data. Chapman and Hall/CRC Press, Boca Raton

17. Dempster AP, Laird NM, Rubin DB (1977) Maximum likelihood from incomplete data via the EM algorithm. J R Stat Soc B 39:1-38

18. Schwarz G (1978) Estimating the dimension of a model. Ann Stat $6: 461-464$

19. Lazarsfeld PF (1950) The logical and mathematical foundations of latent structure analysis. In: Stouffer SA et al (eds) Measurement and Prediction. Princeton University, N.J, pp 362-412

20. McLachlan GJ, Peel D (2000) Finite mixture models. Wiley, New York

21. Birnbaum A (1968) Some latent trait models and their use in inferring an examinee's ability. In: Lord FM, Novick MR (eds) Statistical theories of mental test scores. Addison-Wesley, MA, pp 395-479 
22. McCaffrey DF, Griffin BA, Almirall D, Slaughter ME, Ramchand R, Burgette LF (2013) A tutorial on propensity score estimation for multiple treatments using generalized boosted models. Stat Med 32:3388-3414

23. Elze MC, Gregson J, Baber U, Williamson E, Sartori S, Mehran R, Nichols M, Stone GW, Pocock SJ (2013) Comparison of propensity score methods and covariate adjustment: evaluation in four cardiovascular studies. J Am Coll Cardiol 69:345-357

24. Hernan MA, Brumback B, Robins JM (2001) Marginal structural models to estimate the joint causal effect of nonrandomized treatments. J Am Stat Assoc 96:440-448

25. Joffe MM, Ten Have TR, Feldman HI, Kimmel SE (2004) Model selection, confounder and marginal structural models: review and new applications. Am Stat 58:272-279

26. Rosenbaum PR (2020) Modern algorithms for matching in observational studies. Ann Rev Stat Appl 7:143-176

27. Robins JM (1986) A new approach to causal inference in mortality studies with sustained exposure periods. Application to control of the healthy worker survivor effect. Math Model 7:1393-1512

28. Robins J (1987) A graphical approach to the identification and estimation of causal parameters in mortality studies with sustained exposure periods. J Chron Dis 40:139-161

29. R Development Team (2020) R: a language and environment for statistical computing. R Found. Stat. Comput, Vienna

30. Bartolucci F, Bacci S, Gnaldi M (2017) MultiLCIRT: Multidimensional Latent Class Item Response Theory Models (R package version 2.11)

31. Ahmed J, Khan S, Lim M et al (2012) Enhanced recovery after surgery protocols-compliance and variations in practice during routine colorectal surgery. Colorectal Dis 14:1045-1051

32. Aarts MA, Okrainec A, Glicksman A et al (2012) Adoption of enhanced recovery after surgery (ERAS) strategies for colorectal surgery at academic teaching hospitals and impact on total length of hospital stay. Surg Endosc 26:442-450

33. Hendry PO, Hausel J, Nygren J et al (2009) Determinants of outcome after colorectal resection within an enhanced recovery programme. Br J Surg 96:197-205

34. Vlug MS, Barteles SA, Wind J et al (2012) Which fast track elements predict early recovery after colon cancer surgery? Colorectal Dis 14:1001-1008

35. Poon JT, Fan JK, Lo OS et al (2011) Enhanced recovery programme in laparoscopic colectomy for cancer. Int $\mathrm{J}$ Colorectal Dis $26: 71-77$

36. da Fonseca LM, Profeta da Luz MM, Lacerda-Filho A et al (2011) A simplified rehabilitation program for patients undergoing elective colonic surgery -randomized controlled clinical trial. Int J Colorectal Dis 26:609-616

37. Gianotti L, Beretta S, Luperto M et al (2014) Enhanced recovery strategies in colorectal surgery: is the compliance with the whole program required to achieve the target? Int $\mathrm{J}$ Colorectal Dis 29:329-341
38. ERAS Compliance Group (2015) The impact of enhanced recovery protocol compliance on elective colorectal cancer resection: results from an international registry. Ann Surg 261:1153-1159

39. Gustafsson UO, Hansel J, Thorell A et al (2011) Adherence to the enhanced recovery after surgery protocol and outcomes after colorectal cancer surgery. Arch Surg 146:571-577

40. Gustafsson UO, Scott MJ, Hubner M et al (2019) Guidelines for perioperative care in elective colorectal surgery: enhanced recovery after surgery (ERAS) society recommendations. World $\mathbf{J}$ Surg 43:659-695

41. Rollins KE, Javanmard-Emamghissi H, Lobo DN (2018) Impact of mechanical bowel preparation in elective colo-rectal surgery: a meta-analysis. World J Gastroenterol 24:519-536

42. Gaines S, Shao C, Hyman N et al (2018) Gut microbiome influences on anastomotic leak and recurrence rates following colorectal cancer surgery. Br J Surg 105:131-141

43. Sun Y, Chai F, Pan C et al (2017) Effect of peri-operative goaldirected therapy on postoperative recovery following major abdominal surgery- a systematic review and meta-analysis of randomized controlled trials. Crit Care 21:141

44. Zhuang CL, Ye X, Zhang CJ et al (2013) Early versus traditional postoperative oral feeding in patients undergoing elective colorectal surgery: a meta-analysis of randomized clinical trials. Dig Surg 30:225-232

45. Brower RG (2009) Consequences of bed rest. Crit Care Med $37: 422-428$

46. Block BM, Liu SS, Rowlingson AJ (2003) Efficacy of postoperative epidural analgesia: a meta-analysis. JAMA 290:2455-2463

47. Carli F, Halliday G (1997) Continuous epidural blockade arrests the postoperative decrease in muscle protein fractional synthetic rate in surgical patients. Anesthesiology 86:1033-1040

48. Turi S, Gemma M, Braga M et al (2019) Epidural analgesia vs systemic opioids in patients undergoing laparoscopic colorectal surgery. Int. J. Colorectal Dis. 34:915-921

49. Wijeysundera DN, Pearse RM, Shulman MA et al (2018) Assessment of functional capacity before major non-cardiac surgery: an international, prospective cohort study. Lancet 391:2631-2640

50. Scheede-Bergdahl C, Minnella EM, Carli F (2019) Multi-modal prehabilitation: addressing the why, when, what, how, who and where next? Anaesthesia 74:20-26

51. Smilowitz NR, Oberweis BS, Nukala S (2016) Association between anemia, bleeding, and transfusion with long-term mortality following noncardiac surgery. Am J Med 129:315-323

52. Rubin DB (1979) Using multivariate matched sampling and regression adjustment to control bias in observational studies. J Am Stat Assoc 74:318-328

Publisher's Note Springer Nature remains neutral with regard to jurisdictional claims in published maps and institutional affiliations. 